\title{
RESEARCH
}

\section{Optimization Strategy of Wireless Charger Node Deployment Based on Improved Cuckoo Search Algorithm}

\author{
Yang Wang, Feifan Wang, Yujun Zhu*, Yiyang Liu and Chuanxin Zhao
}

\author{
${ }^{*}$ Correspondence: \\ zhuyujun@mail.ahnu.edu.cn \\ Anhui Normal University, School \\ of Computer and Information, \\ China, Wuhu \\ Full list of author information is \\ available at the end of the article
}

\begin{abstract}
In wireless rechargeable sensor network, the deployment of charger node directly affects the overall charging utility of sensor network. Aiming at this problem, this paper abstracts the charger deployment problem as a multi-objective optimization problem that maximizes the received power of sensor nodes and minimizes the number of charger nodes. First, a network model that maximizes the sensor node received power and minimizes the number of charger nodes is constructed. Second, an Improved Cuckoo Search (ICS) algorithm is proposed. This algorithm is based on the traditional Cuckoo Search algorithm (CS) to redefine its step factor, and then use the mutation factor to change the nesting position of the host bird to update the bird's nest position, and then use ICS to find the ones that maximize the received power of the sensor node and minimize the number of charger nodes optimal solution. Compared with the traditional cuckoo search algorithm and multi-objective particle swarm optimization algorithm, the simulation results show that the algorithm can effectively increase the receiving power of sensor nodes, reduce the number of charger nodes and find the optimal solution to meet the conditions, so as to maximize the network charging utility.

Keywords: Wireless Rechargeable Sensor Network; Node Deployment; Deployment Optimization; Cuckoo Search
\end{abstract}

\section{Introduction}

With the rapid development of microelectronics technology, signal processing technology, wireless communication technology and computer networks, wireless sensor networks have emerged. Wireless Sensor Network (WSN) is a multi-hop selforganizing network system formed by wireless communication. The sensor node cooperatively senses, collects, and processes information of the perceived object in the network coverage area and sends it to the observer[1, 2]. Wireless sensor networks are widely used in many important fields such as forest fire detection, animal tracking, military area monitoring, early earthquake detection, and border monitoring due to their low cost, low power consumption and multi-function[3, 4, 5, 6, 7].But as the size of the network increases, it becomes increasingly difficult and unrealistic to periodically replace batteries for all nodes, and limited battery energy will eventually lead to limited network life. In order to solve the problem of limited life in wireless sensor networks, many scholars at home and abroad have conducted a lot of research, and the solutions can be divided into three categories: node energy saving, natural energy collection and wireless charging. The energy-saving method 
mainly reduces the energy loss per unit time by compressing the transmitted data packets, clustering the network, and selecting the dynamic cluster head. The natural energy harvesting method requires the use of an energy converter on the node through which it can harvest energy (such as solar energy, wind energy, etc.) from the natural environment to extend its life. However, obtaining energy from the natural environment will bring uncertainty in the energy source (such as day and night, strong winds and weak winds), and the energy conversion efficiency is not high. The wireless charging method refers to providing the network with a wireless charging source (such as a static charging station, a mobile charging car, etc.), the charging source travels in the network according to the charging trajectory, and charges the nodes in the network during the driving process. Comparing the energy harvesting and wireless charging technologies of the natural environment, although both charging methods can ensure that the sensor nodes work persistently, the wireless charging method has better performance in the stability of the charging source and the predictability of the energy acquired by the nodes. excellent. So the academic community proposed the Wireless Rechargeable Sensor Network (WRSN)[8, 9].In the WRSN, the sensor node energy comes from the charger node instead of the capacity-limited battery. The nodes in the wireless charging sensor network rely on wireless energy transmission technology to obtain energy. The transmitting end of the wireless energy transmission system converts the electrical energy into electromagnetic waves for transmission, and the receiving end receives the electromagnetic wave energy and converts it into electrical energy. Changes in energy sources increase the reliability, flexibility, and scalability of sensor networks, but a key issue in WRSN is the deployment of wireless chargers, which are expensive and require a lot of time and cost to deploy. How to effectively deploy the wireless charger to minimize the charging cost of the network is an urgent problem to be solved. The main contributions of this paper are as follows:

1) We first proposed a network model that maximizes the sensor node received power and minimizes the number of charger nodes. By maximizing the sensor node received power and minimizing the number of charger nodes, the overall charging efficiency of the network is improved.

2) This paper presents a new algorithm: Improved Cuckoo Search (ICS). The algorithm redefines its step factor on the traditional cuckoo search algorithm, and then uses the mutation factor to change the nesting position of the host bird, thereby updating the bird's nest position to generate new populations.

3) The proposed model is optimized by the Improved Cuckoo Search (ICS). Experiments show that the algorithm is superior to other algorithms in terms of charging efficiency.

\section{Related work}

At present, researchers have proposed a lot of deployment strategies for charger nodes. Zhu. X et al.[10] consider sensor node energy harvesting to deploy nodes, and achieve optimal target coverage through heuristic algorithms. Yang. C et al.[11] proposed the minimum energy collection node position model of energy neutral coverage and connection. Under the premise of approximation algorithm, the target node is monitored by different energy harvesting rates of sensor nodes, thus achieving target coverage. Li. S et al.[12] used the concept of wireless charger to charge 
the sensor. The main purpose was to deploy a minimum number of wireless chargers to charge all the sensors. Then a greedy cone coverage algorithm and an adaptive coverage algorithm were proposed to make the charger deployed at the most. Good grid points to cover the sensor, experimental results show that the greedy cone coverage algorithm is better than the adaptive cone coverage algorithm. Chen et al.[13] used the Particle Swarm Optimization (PSO) algorithm to alleviate the problem of charger deployment. First, the charger was randomly deployed, and then the charging position and charging direction were changed by PSO to determine the optimal solution. Yu. $\mathrm{N}$ et al.[14] given a certain number of directional wireless chargers and candidate locations, determine the placement position and direction angle of each charger under the connection constraints of the wireless charger, so as to maximize the overall charging utility. In addition, there are also literatures that consider the charging characteristics of chargers or devices[15, 16].

This paper proposes to maximize the received power of the sensor node and minimize the number of charger nodes, and has developed a multi-objective optimization problem to achieve this goal. Group intelligent optimization algorithm[17] is an effective method to solve multi-objective optimization problems. The particle swarm optimization algorithm PSO based on bird foraging behavior[18] is used to solve the multi-objective optimization problem, however, its performance is significantly affected by the initial value of the parameter[19].The Invasive Weed Optimization (IWO) algorithm is proposed in Ref.[20]. IWO is inspired by the phenomenon that is common in agriculture, the colonization of invasive weeds, and is easy to implement. However, the performance of IWO is also affected by the choice of parameters. The Group Wolf Optimization algorithm (GWO)[21] is inspired by the hunting of prey in the wolves in nature, which simulates the social level of the wolf, thus achieving a higher convergence rate. However, GWO's global search capabilities are weak. The bat algorithm (BA)[22] is based on the echo localization behavior of bats and generates new solutions through random flight, thereby improving the ability of local search. However, due to the lack of mutation mechanism of BA individuals, the accuracy of BA is low in some applications. Reference[23] proposed a new algorithm adaptive levy flower pollination algorithm (ALFPA), which combines the Levy fight mechanism and the flower pollination algorithm of CS. The convergence speed of the algorithm is improved by introducing additional components.

In order to improve the overall performance of the network, some efficient algorithms are also used in it. Guo. P et al.[24] proposed a heuristic algorithm based on the greedy algorithm to deploy static chargers to minimize the charging time of sensor nodes. In reference[25], a method based on the improved maxmin ant system is proposed to optimize the energy constraints of mobile wireless chargers (MWC$\mathrm{S})$, aiming to minimize the total energy consumption in the charging cycle and the equalization strategy under the limited energy constraints of MWCS. Chien. W et al.[26] Proposed a layered algorithm based on simulated annealing algorithm to deploy indoor sensor nodes to control factory production, improve production quality, and optimize the wireless rechargeable sensor network. In reference[27], Chen. Z et al. proposed a delay fault-tolerant mobile charging scheduling scheme (DMCSS), designed an efficient next charging node selection algorithm, in the case of limited mobile charger capacity, to maximize the charging efficiency and prevent premature 
node failure. So as to optimize the charging track and improve the charging efficiency. Tomar. A et al.[28] proposed a charging strategy based on a gravitational search algorithm, which uses a mobile charger to charge sensor nodes to solve the problem of node starvation, thereby improving network performance. From the above analysis, we can see that the previous work used a single meta-heuristic method to solve the problem. Compared with the previous method, this paper considers that the cuckoo search algorithm has weak local search ability and lacks a mutation mechanism, so the step size control factor is redefined, and then use the mutation factor to change the nesting position of the host bird, thereby balancing the local search and global search capabilities, not only maintaining the diversity of the population, but also expanding the sampling space, preventing the loss of the best individual, and improving the algorithm Convergence speed and robustness.

In this paper, the charger deployment problem is abstracted as a multi-objective optimization problem that maximizes sensor node reception power and minimizes the number of charger nodes. Through ICS to find the optimal solutions to maximize the receiving power of the sensor node and minimize the number of charger nodes, the simulation results show that the effectiveness of the algorithm is superior to other comparison algorithms in accuracy and convergence speed.

\section{System model}

\subsection{Problem description}

In a wireless rechargeable sensor network, it is assumed that there are $\mathrm{S}$ chargeable sensors distributed in a two-dimensional area, and the positions of the sensors are known. There are $\mathrm{M}$ omnidirectional wireless chargers to be arranged, each of which can be placed anywhere in the area and can be oriented arbitrarily. Assuming that the charging area of each charger covers a subset of the sensors, adjacent chargers can cover a common sensor, which means that a sensor may be simultaneously covered by multiple chargers for charging. As shown in Figure.1.

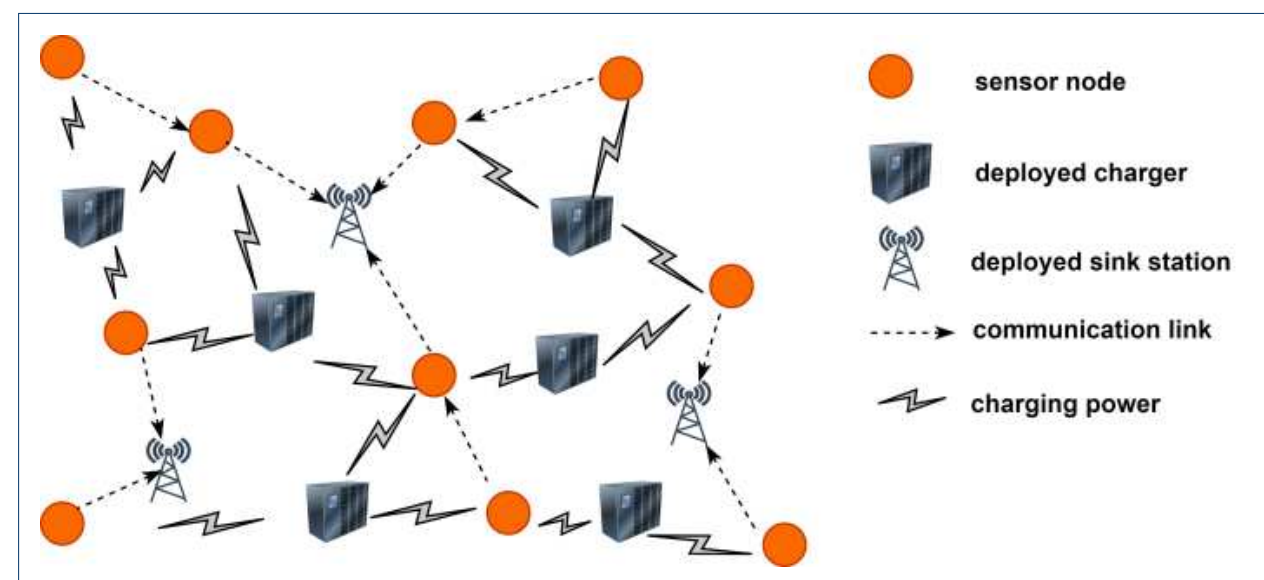

Fig.1 Deployment of wireless rechargeable sensor nodes

\subsection{System model}

As can be seen from Figure 1, the wireless rechargeable sensor network is composed of sensor node set $S=\left\{S_{1}, S_{2}, \ldots, S_{N}\right\}$ and wireless charger node set 
$C=\left\{C_{1}, C_{2}, \ldots, C_{K}\right\}$. The location of the sensor node is known, the wireless charger can be moved to any location, but it cannot be moved after deployment. The wireless charger is powered by solar energy, the energy is not limited, and any sensor node can be charged.

The charging model we use is related to the distance between the sensor and the charger. According to this model[29], $\mu$ is defined as the charging efficiency.

$$
\mu=\frac{\mathrm{G}_{\mathrm{s}} \mathrm{G}_{\mathrm{r}} \eta}{L_{p}}\left(\frac{\lambda}{4 \pi(d+\beta)}\right)^{2}
$$

Among them, $d$ is the distance between the sensor node and the charger node, $G_{s}$ is the source antenna gain, $G_{r}$ is the receiving antenna gain, $L_{p}$ is the polarization loss, $\lambda$ is the wavelength, $\eta$ is the rectification efficiency, and $\beta$ is the adjustment model parameter for short-distance propagation. In formula (1) except $d$, all others are constants, so it is simplified to

$$
\mu=\frac{\alpha}{(d+\beta)^{2}}
$$

Here, $\alpha$ represents other parameters in formula (1), including $G_{s}, G_{r}, L_{p}, \lambda, \eta$. Therefore, the received power of each sensor node $S_{i}$ is

$$
P_{i}=\sum_{n=1}^{N} \mu_{n} \cdot P_{0}
$$

In Equation(3), $P_{0}$ is the source power of the charger node and $\mu_{n}$ is the power of the $n_{\text {th }}$ charger node.

Then, in order to achieve the goal of improving the received power of the sensor node, the minimum power of the sensor node is maximized.

$$
F_{1}=\max \left[\min P_{i j}\right]
$$

Here, $P_{i j}$ is the charging power of the $i_{t h}$ charger node to the $j_{t h}$ sensor node. $i \in$ $[0, K], j \in[0, N]$. For ease of understanding, formula (4) can be normalized,namely

$$
F_{1}=\min \left[\max \left(\frac{P_{w o r s t}}{P_{i j}}\right)\right]
$$

In Equation(5), $P_{w}$ orst indicates that the charging efficiency is at the upper limit of the charging range and the range ratio is within $[0,1]$.

The maximum number of sensor node coverage is achieved with a minimum of charger nodes, and to accomplish this goal, the number of charger nodes deployed is critical. Suppose $F_{\max }$ is the total number of charger nodes and Fred is the amount of redundancy of the charger node, then

$$
F_{2}=F_{\max }-F_{\text {red }}
$$




\subsection{Improved cuckoo search algorithm}

\subsubsection{Basic principles of cuckoo search algorithm}

Cuckoo Search (CS)[26], proposed by Professor Yang in 2009, is a heuristic swarm intelligence algorithm. This algorithm mainly simulates the behavior of cuckoos to find bird nests and place eggs, combined with Lévy The bird's nest position is updated in flight to complete the update of each generation. If the updated position is better than the current position, the bird's nest position is updated, otherwise the current position is retained. The cuckoo's path and location update formula for searching the bird's nest by Lévy flight mode is as follows:

$$
x_{i}^{(t+1)}=x_{i}^{(t)}+\alpha \oplus \operatorname{Lévy}(\lambda)
$$

In Equation $(7), x_{i}^{(t)}$ and $x_{i}^{(t+1)}$ represent the bird's nest positions of the $t_{t h}$ and $t+1_{t h}$ generations respectively, $\alpha$ represents the step size control factor, $\oplus$ is a point-to-point multiplication, Lévy ( $\lambda$ random search path, and the relationship with time $t$ follows the Lévy distribution, namely:

$$
\operatorname{Lévy}(\lambda) \sim \mu=\mathrm{t}^{-\lambda}(1<\lambda \leq 3)
$$

In Equation(7), the size of $\alpha$ is related to the search domain of the problem. When $\alpha$ is large, the algorithm's walk length will be relatively large, so that the largescale search domain can be explored more effectively. Usually $\alpha$ is taken as $o(1)$. In order to make the algorithm have faster convergence ability, the difference between different solutions can be introduced to make the algorithm have a certain variable step size ability, then $\alpha$ can be expressed as equation (9):

$$
\alpha=\alpha_{0}\left(x_{j}^{(t)}-x_{i}^{(t)}\right)
$$

Where $\alpha_{0}$ is a constant, generally 0.01 .

Studies have shown that the use of Lévy flight search mechanism in intelligent algorithms can expand the search range and increase population diversity. In order to facilitate calculation, the literature[30] uses the following formula to calculate the Lévy random number:

$$
\operatorname{Lévy}(\lambda)=\frac{\varphi \times \mu}{|v|^{1 / \beta}}
$$

Among them, $\mu$ and $v$ follow the standard normal distribution, $\beta$ is a constant, the value is $[1,2]$, the value of $\varphi$ is as follows:

$$
\varphi=\left\{\frac{\Gamma(1+\beta) \times \sin \left(\frac{\pi \times \beta}{2}\right)}{\Gamma\left[\left(\frac{1+\beta}{2}\right) \times \beta \times 2^{\frac{\beta-1}{2}}\right]}\right\}^{1 / \beta}
$$

Combined with formulas $(7) \sim(11)$, the new solutions generated during Lévy's flight are as follows:

$$
x_{i}^{(t+1)}=x_{i}^{(t)}+\alpha_{0} \frac{\varphi \mu}{|v|^{1 / \beta}}\left(x_{i}^{(t)}-x_{b e s t}\right)
$$


Among them, $x_{\text {best }}$ represents the current optimal solution. After the location update, use the random number $r \in[0,1]$ to compare with $P_{a}$ ( $P_{a}$ is the probability that the host bird finds alien eggs, and the value is [0,1]). If $r>P_{a}$, set $x_{i}^{(t+1)}$ preference random walks are used to generate the same number of new solutions. Preference random walks are shown in equation (13):

$$
x_{i}^{(t+1)}=x_{i}^{(t)}+r\left(x_{j}^{(t)}-x_{k}^{(t)}\right)
$$

In the formula, $r$ is the compression factor, which is a uniformly distributed random number in the interval $[0,1], x_{j}^{(t)}$ and $x_{k}^{(t)}$ represent the two random solutions of the $t_{t h}$ generation.

\subsubsection{Improved cuckoo search algorithm}

Aiming at the problem that the cuckoo search algorithm solves multi-objective complex problems, local optimal solutions and low accuracy are prone to appear. This paper proposes an improved cuckoo search algorithm (ICS). Based on the original algorithm, this algorithm updates the cuckoo search area by redefining its step control factor, then

$$
\alpha=\frac{\left|x_{i}^{(t)}-x_{\text {best }}^{(t)}\right|}{d_{\max }}
$$

In formula (15), the new solution is generated by it, and the moving step of the solution is composed of two parts, one is the generated random number $r$, and the other is the vector difference operation $x_{j}^{(t)}-x_{k}^{(t)}$. However, there is a problem in updating the solution in this way. The generation of the new solution is random, and the updating of the solution has no directionality.Therefore, the mutation factor $\theta$ is introduced in the local search, which makes the directivity of the solution more random and enhances the diversity of the descendant population[31].namely

$$
x_{i}^{(t+1)}=x_{i}^{(t)}+\theta\left(x_{j}^{(t)}-x_{k}^{(t)}\right)
$$

In Equation(15), $\theta$ is the variation factor and $\theta \in N(0,1)$ is a random vector subject to a normal distribution.

The working flow chart of the improved cuckoo search algorithm is shown in Figure 2.

\subsubsection{Multi-objective optimization}

The optimal solution selection and ranking method based on Pareto dominance: select a bird's nest $x_{i}$ in the bird's nest group and compare it with the remaining bird's nest in turn, and divide the population into two parts according to the relationship between the remaining bird's nest in the bird's nest group and $x_{i}$. The bird's nest not related to $x_{i}$, part $B$ is the bird's nest dominated by $x_{i}$. If $x_{i}$ is not dominated by any bird's nest, store $x_{i}$ in an external set that holds the Pareto solution set. Then repeat the above process for the bird nest in part $A$ until the bird nest in part $A$ is emptied. 


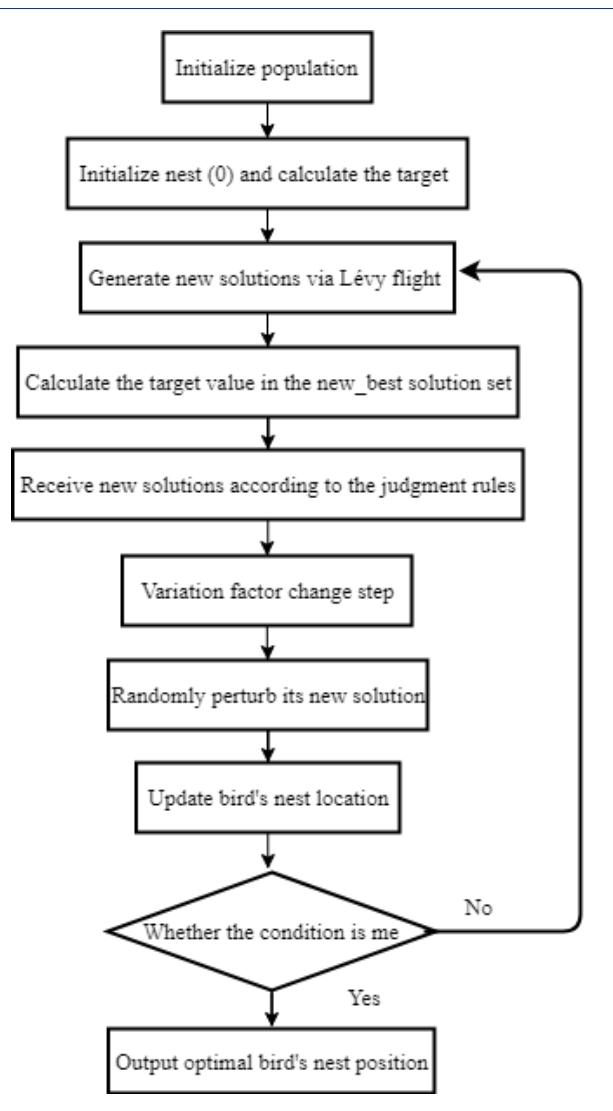

Fig.2 Workflow of improved cuckoo search algorithm

The adaptive weighted fitness allocation is used to solve the objective function values of each nest in the target space, and the weight values of each objective function are obtained by comparison.

$$
\lambda_{j}=\frac{1}{f_{j}^{\max }-f_{j}^{\min }}
$$

Then the fitness value of the nest is

$$
F i t\left(x_{i}\right)=\lambda_{1}\left(F_{1}^{\max }-F_{1}\left(x_{i}\right)\right)+\lambda_{2}\left(F_{2}^{\max }-F_{2}\left(x_{i}\right)\right)
$$

The Pareto solution set can be distinguished by the fitness value. In the Pareto solution set of the external set, two solutions are randomly selected to compare the fitness values, and then the better solution is selected as $p_{\text {besti(t) }}$ or $g_{\text {besti(t) }}$. If the current nest does not satisfy the constraint, the nest is deleted and a new nest is randomly generated to increase the diversity of the progeny population.

\subsubsection{Algorithm implementation}

The charger deployment problem in this paper is divided into two sub-problems: maximizing the received power of the sensor nodes and minimizing the number of 
charger nodes, and then searching for multiple targets by optimizing these two subproblems based on the improved cuckoo search algorithm. The optimal solution set makes it better to approach the Pareto frontier. The algorithm is as follows:

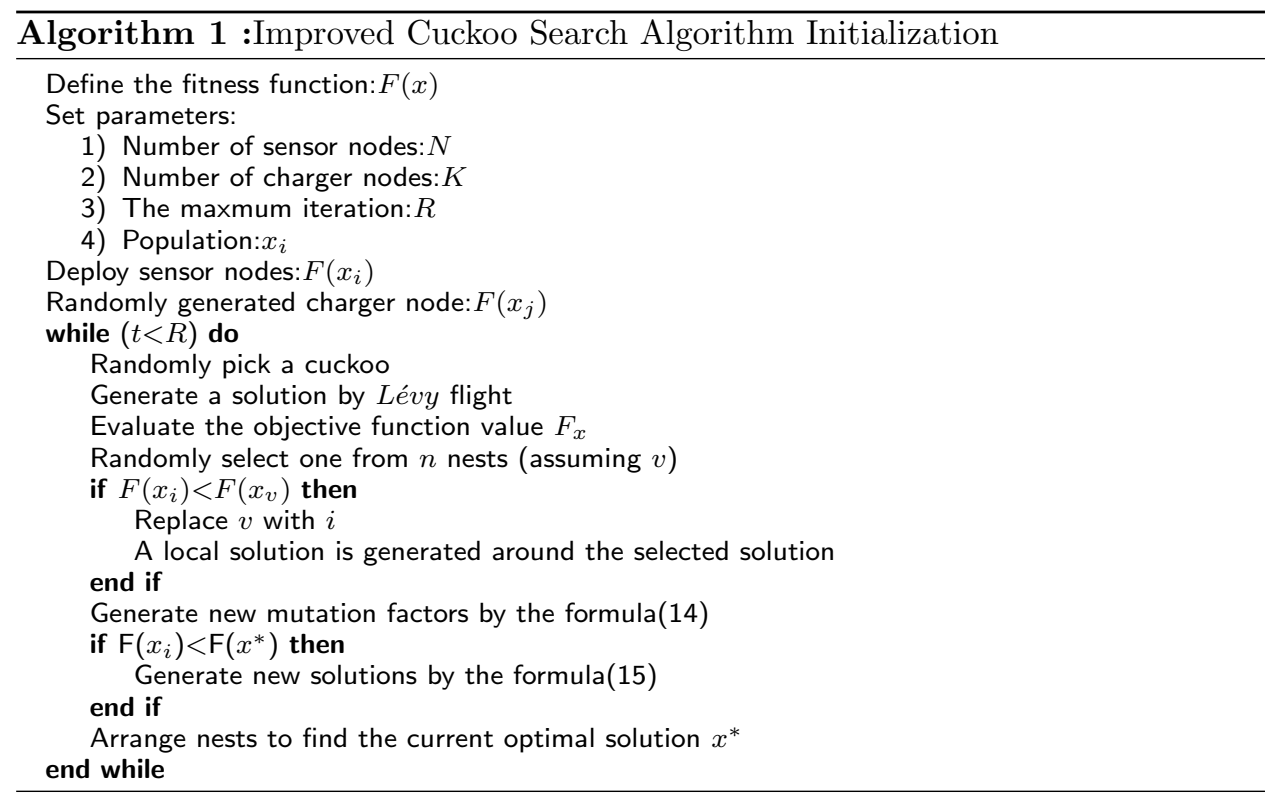

According to the above algorithm 1, the coordinates of the sensor node and the charger node are initialized, the optimal solution is searched through iteration, and then a local new solution is generated by Lévy flight around the optimal solution, and then the new solution is generated by updating the position of the bird's nest. If the new solution does not satisfy the condition, the search is iterated again, and this process strengthens the local search. By constantly moving the position of the charger node, the received power of the sensor node is calculated, so as to minimize the number of charger nodes. Such as algorithm 2.

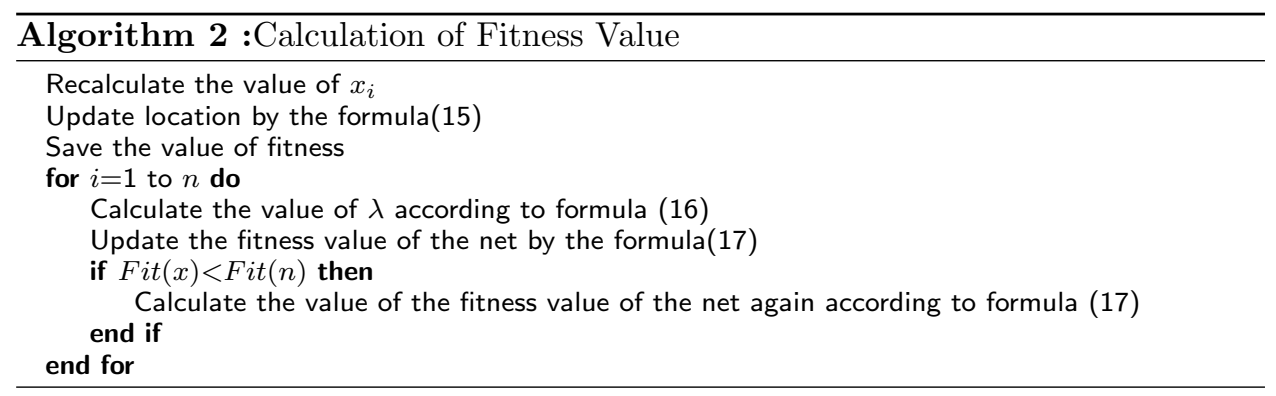

In the ICS algorithm, in order to facilitate calculation, the size of the bird nest and the merge set are set to $M$, the number of targets for multi-objective optimization is $N$, and the dimension is $D$. According to the flow of the algorithm, the analysis of the time complexity of the main steps of the ICS algorithm is as follows.

In the first step, the initialization complexity is $O(M D)$; in the second step, the effective solution is selected from the bird's nest to enter the merged set, and its complexity is $O\left(M N^{2}\right)$. Secondly, when performing maintenance strategies, it is necessary to calculate the degree of convergence, the degree of distribution, and 
the auxiliary judgment index respectively. Its time complexity is $O\left(N M^{2}\right)$, and the complexity of multi-index ranking is $O(M \log M)$. In the third step, the position of the bird's nest Update. In summary, the maximum time complexity of the algorithm in this paper is $O\left(M N^{2}\right)$.

\section{Experimental results and analysis}

\subsection{Parameter settings}

This section verifies the performance of the proposed algorithm through simulation experiments. Specifically, the multi-objective particle swarm optimization algorithm (MOPSO), the cuckoo search algorithm (CS) and the improved cuckoo search algorithm algorithm proposed in this paper are compared. The changes of the received power of the sensor nodes, the number of charger nodes, the power of each sensor node and the optimal solution set distribution in the given plane area of these three algorithms are studied. As shown in Table 1, the basic parameter settings of the simulation experiment are given[15].

Table 1: Basic parameter setting

\begin{tabular}{cc}
\hline Parameter & Defaults \\
\hline Geographic range & $100 \mathrm{~m} \times 100 \mathrm{~m}$ \\
Number of sensor nodes & 100 \\
Parameters in the charging model: $\alpha$ & 40 \\
Parameters in the charging model: $\beta$ & 100 \\
Source power of the charger node & 40 \\
\hline
\end{tabular}

Among them, the number of charger nodes is set up to 40 . On the basis of 40 charger nodes, the number of charger nodes is optimized by ICS to minimize the number of charger nodes.

\subsection{Experimental results and analysis}

The cuckoo search algorithm, multi-objective particle swarm optimization algorith$\mathrm{m}$ and the improved cuckoo search algorithm proposed in this paper compare the received power of sensor nodes. It can be seen from Figure 3 that after 100 iterations, ICS is always better than CS and MOPSO. Maximize the received power of the sensor node to find the optimal solution that satisfies the condition. The comparison results show that the ICS is $30.4 \%$ higher than that of CS and $25 \%$ higher than MOPSO. Therefore, the improved cuckoo search algorithm with better global and local search ability is introduced into the sensor node receiving power. It can effectively reduce the error of the receiving power of the sensor node, and accelerate the solution speed of finding the optimal solution problem. It is an intelligent algorithm with high precision and fast speed.

Within the specified sensing domain, sensor nodes are randomly distributed and charged by deploying charger nodes, reducing the number of charger nodes to reduce costs. In this paper, the ICS algorithm is used to deploy the charger node. It can be seen from Figure 4 that after 90 iterations, the number of charger nodes obtained by using ICS gradually approaches an optimal value, and the number of charger nodes requested by MOPSO and CS is constantly changing,but did not approach a certain value, and MOPSO and CS seek the number of charger nodes is still changing continuously and does not approach a certain value. Experimental data shows that 


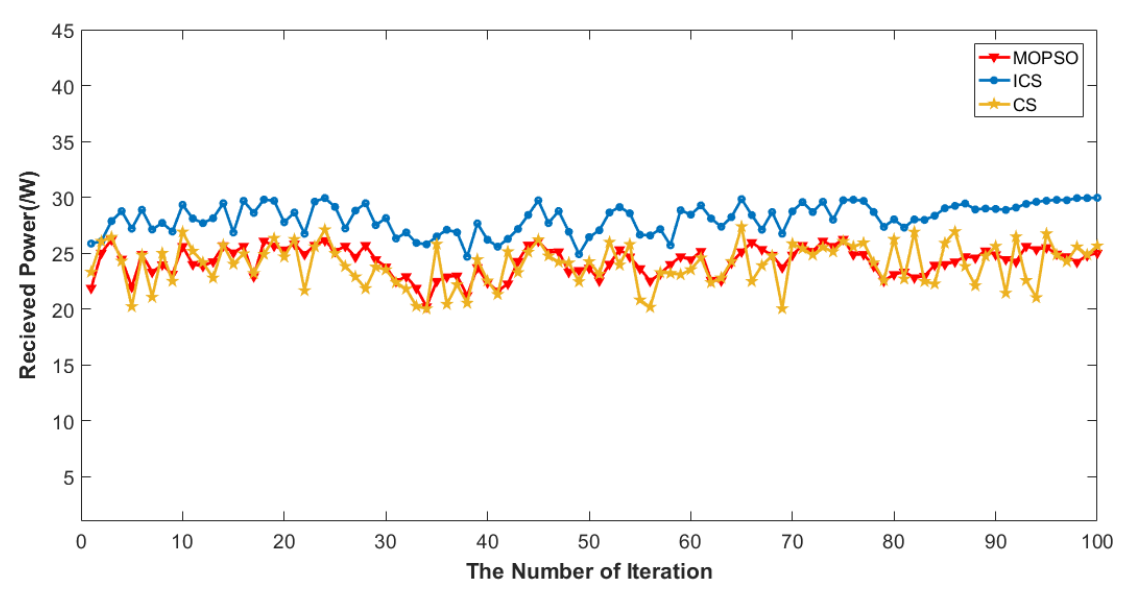

Fig.3 Comparison of algorithms for receiving power of sensor nodes

ICS is $53.3 \%$ lower than CS and $30.3 \%$ lower than MOPSO, so the number of charger nodes is reduced. ICS is looking for the optimal number of charger nodes than MOPSO and CS, therefore, ICS minimizes the number of charger nodes.

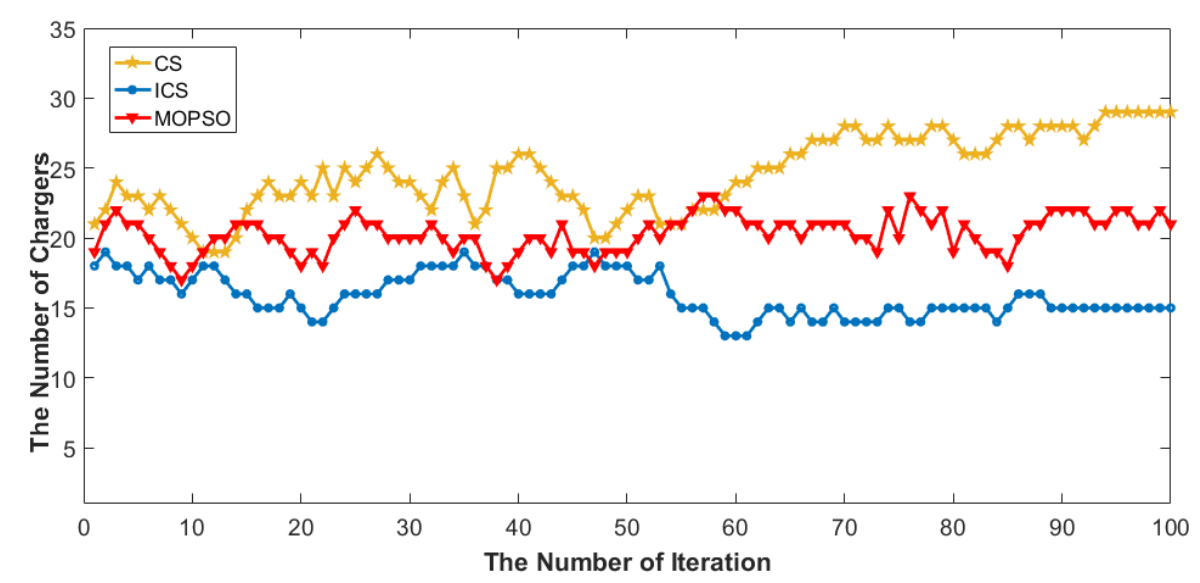

Fig.4 Algorithm comparison of the number of charger nodes

Sensor nodes are effectively charged and have a certain amount of energy to better collaborate and perceive each other data information. In this paper, the received power of the sensor node is an optimization goal. Distribute 100 sensor nodes in the sensing field, so that each other can better transfer data between each other, and collect the received power of each sensor node through the ICS algorithm. As shown in Figure 5, it can be seen that the distribution of 100 sensor nodes makes The received power distribution is more uniform and concentrated, which is more helpful for the subsequent experiments, and it is easier to achieve the maximum power of the sensor node.

In this paper, ICS continuously moves and updates the position of charger nodes to determine the best position and number of charger nodes, so as to achieve the 


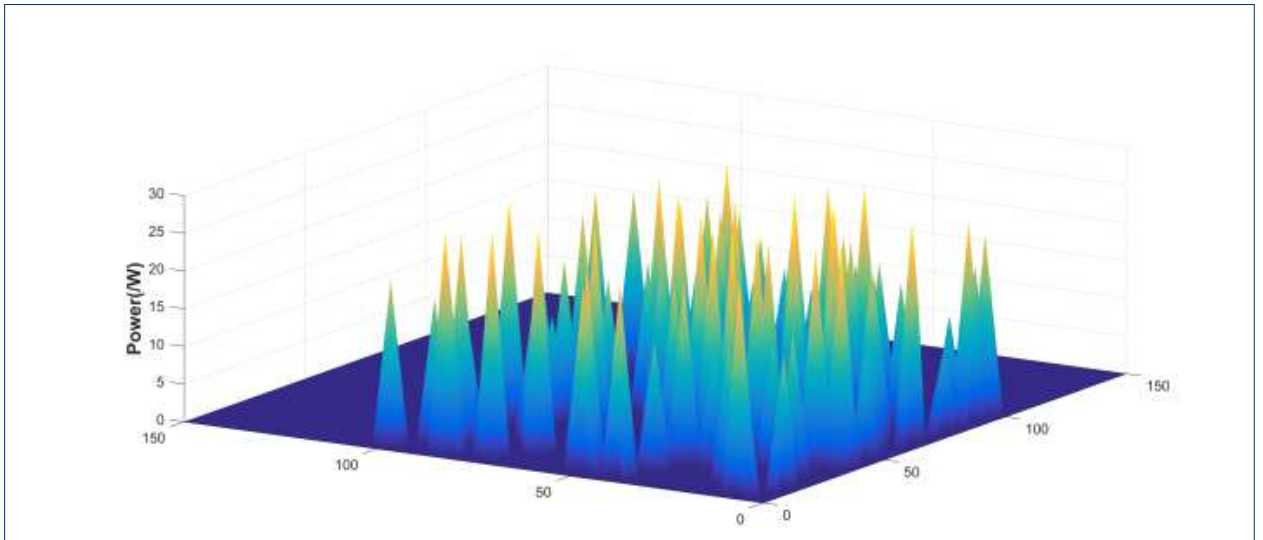

Fig.5 The received power of the 100 sensor nodes

goal of minimizing the number of charger nodes.Figure 6 is the deployment diagram of the charger node's constantly updated position.

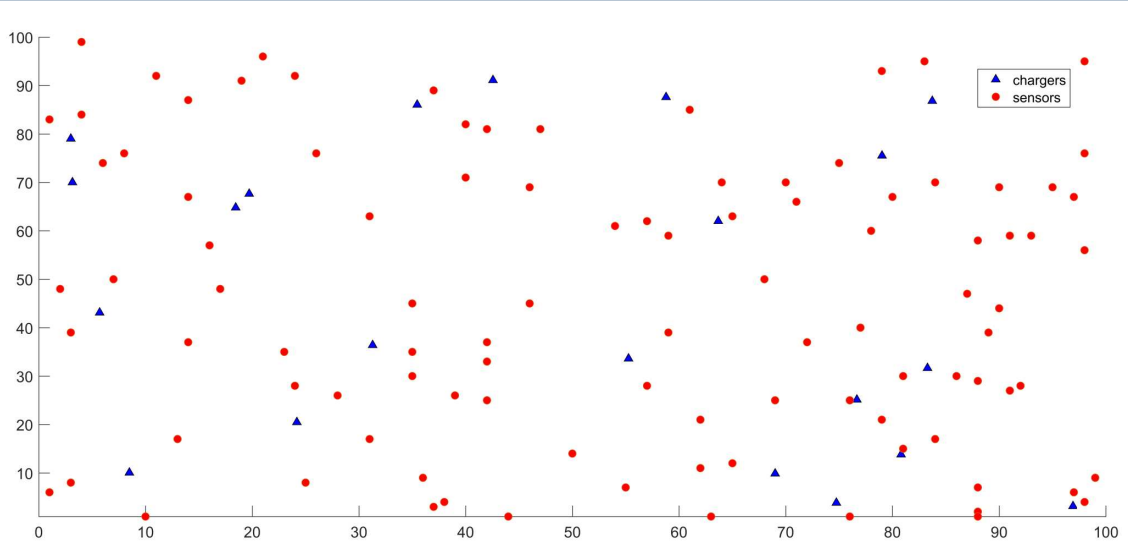

(a)

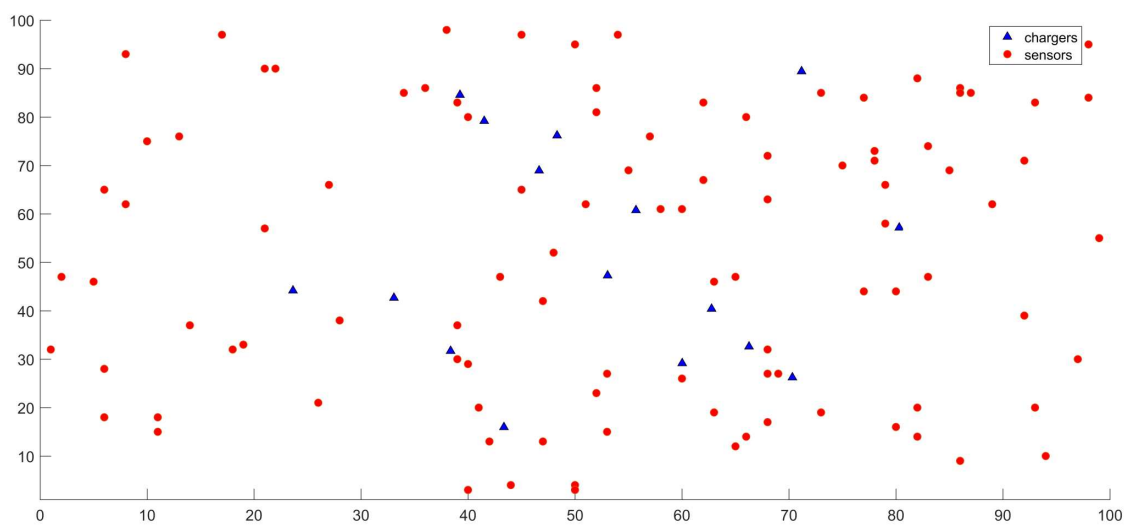

(b)

Fig.6 Charger node deployment 
The ICS is used to solve the received power of the sensor node and the number of charger nodes, so that the two can better approach the optimal solution. It can be seen from Figure7 that after 100 and 200 iterations, the non-inferior solution set of the maximum received power of the sensor node and the minimum number of charger nodes is gradually close to the Pareto frontier, and the solution distribution obtained is concentrated and uniform. It can also be seen from the figure that using the ICS algorithm to solve the multi-objective problem can converge to the better solution more effectively and accurately.

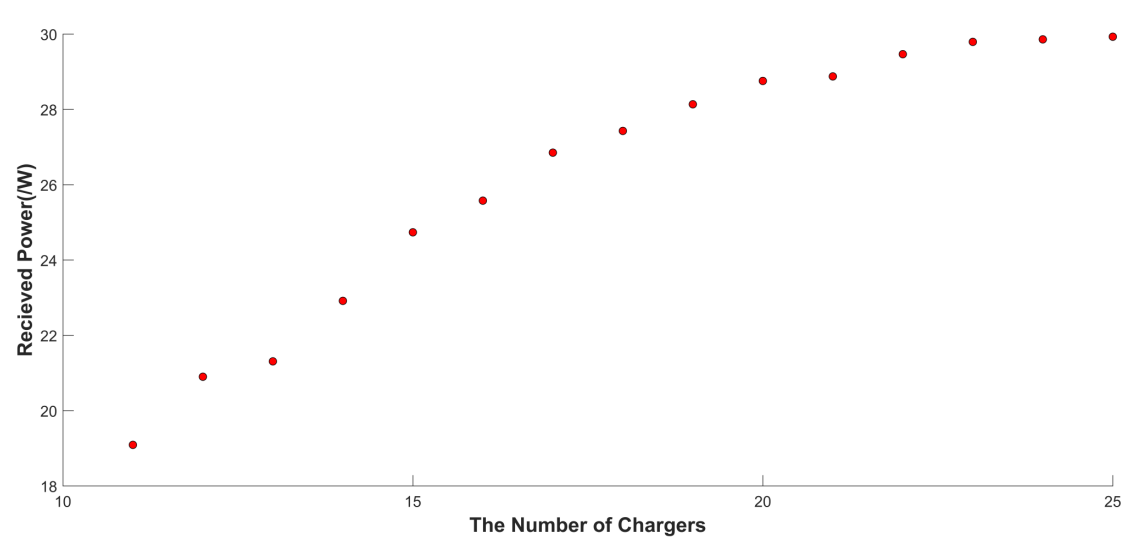

(a)

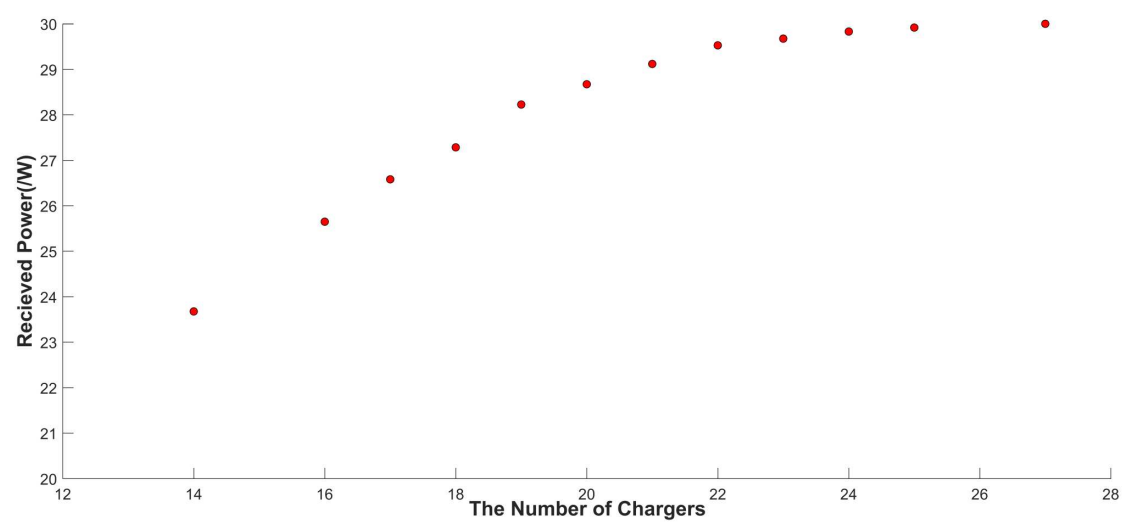

(b)

Fig.7 Pareto solution sets with different iteration time

Through Figure 8, the fitness value obtained after 100 iterations shows that although the ICS has outliers, the data distribution is concentrated and uniform, and the convergence is stable, which makes the solution set obtained by ICS closer to Pareto. cutting edge. In contrast, the data distribution in MOPSO is more dispersed and uneven, and the data in CS is more discrete, and the solution in the data concentration part does not approach the fitness value.

Through the Pareto frontier, ICS, MOPSO and CS are observed to approach the optimal solution, as shown in Figure 9. After many iterations, the multi-objective problem is solved by the ICS algorithm. The solution distribution obtained by ICS is relatively uniform, and the convergence speed is fast and approaches Pareto frontier 


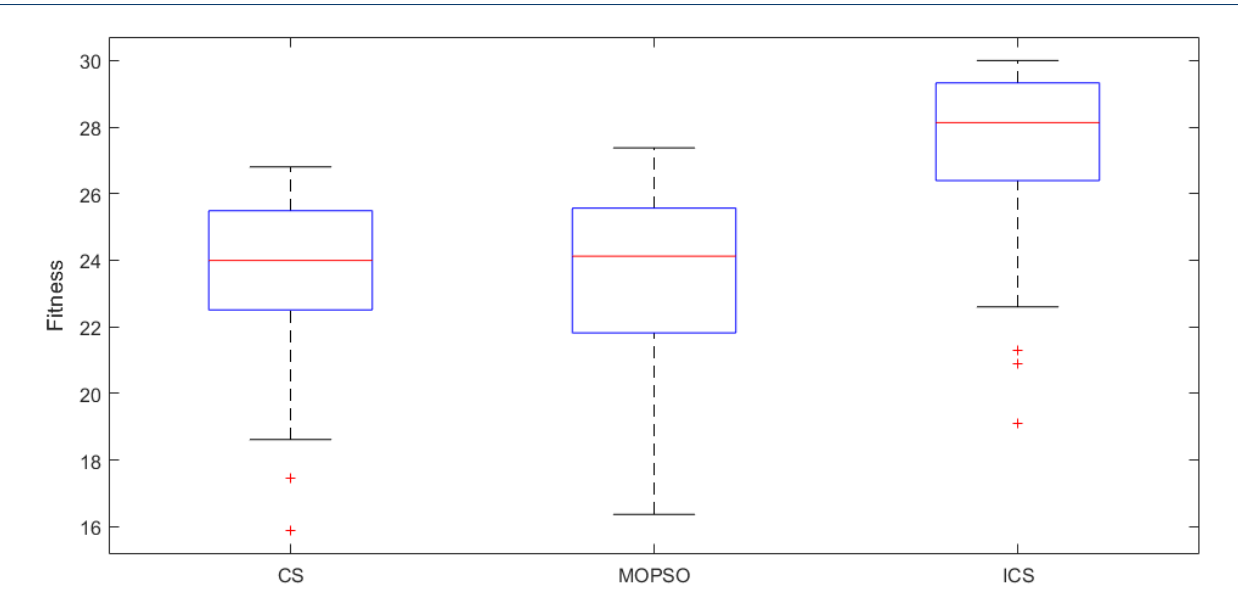

Fig.8 Statistical box graph of fitness value under 100 iterations

earlier. In comparison, the solution distribution obtained by MOPSO and CS is more discrete and the convergence speed is slower. After the experimental comparison, it shows that the convergence speed of ICS is $45 \%$ higher than that of CS and $21.4 \%$ higher than that of MOPSO. Therefore, ICS can converge more effectively and accurately to the better solution than MOPSO and CS, and converges.

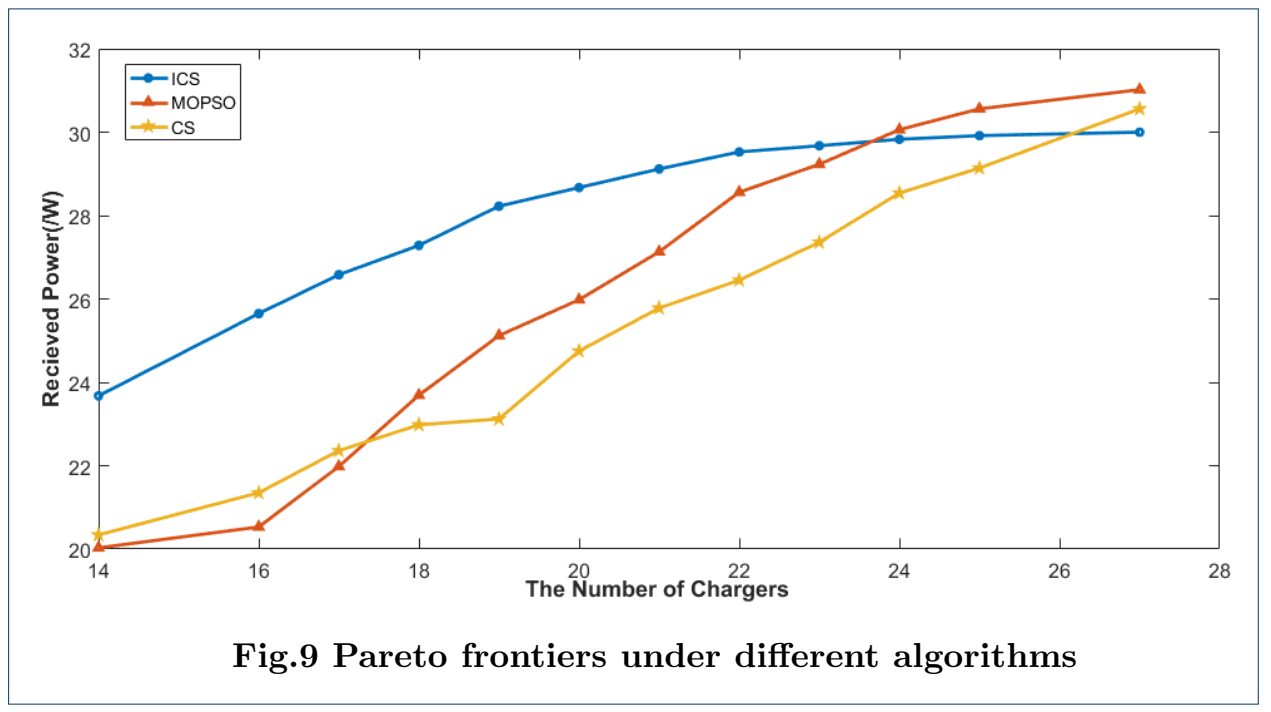

\section{Conclusion}

This paper focuses on the sensor node receiving power and charger node deployment, and optimizes the charging of the entire network by optimizing the deployment of the charger node. Using the improved cuckoo search algorithm, multi-objective optimization is achieved by maximizing the received power of the sensor nodes and minimizing the number of charger nodes to find the optimal solution that satisfies the multi-objective problem. In addition, the correctness and effectiveness of the proposed model and algorithm are verified. The experimental results show that the proposed algorithm (ICS) is superior to other comparison algorithms in terms of 
accuracy and convergence speed compared with other algorithms, and it is also improved. In future research, the problem of the energy loss of the charger node during the charging process will be considered.

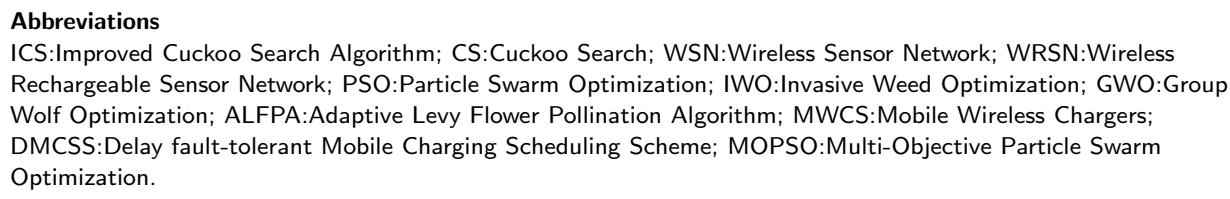

\section{Acknowledgements}

The authors acknowledged the anonymous reviewers and editors for their efforts in valuable comments and suggestions.

\section{Author's contributions}

Y.Wang proposes the innovation ideas and theoretical analysis, and F.Wang carries out experiments and data analysis.Y.Zhu, Y.Liu and C.Zhao conceived of the study, and participated in its design and coordination and helped to draft the manuscript. All authors read and approved the final manuscript.

\section{Funding}

This research was supported by the National Natural Science Foundation of China (61871412) and the Anhui Provincial Natural Science Foundation (1908085MF214).

\section{Availability of data and materials}

Data sharing is not applicable to this article as no data sets were generated or analyzed during the current study.

Competing interests

The authors declare that they have no competing interests.

Author details

Network of Internet of Things Technology and Application Laboratory of Anhui Normal University, Anhui Province.

\section{References}

1. Soldani, D., Manzalini, A.: Horizon 2020 and Beyond: on the 5G Operating System for a True Digital Society. IEEE Vehicular Technology Magazine 10, 32-42 (2015)

2. Wang, B., Wang, K., Lu, Z.: Comparison Study of Non-Orthogonal Multiple Access Schemes for 5G. Proceedings of IEEE International Symposium on Broadband Multimedia Systems and Broadcasting, pp. 1-5 (2015).

3. GarciaJimenez, S., Jurio, A., Pagola, M., DeMiguel, L., Barrenechea, E., Bustince, H., Forest Fire Detection: A Fuzzy System Approach based on Overlap Indices. Applied Soft Computing 52, 834-842 (2016)

4. Dominguezmorales, J.P., Riosnavarro, A., Dominguezmorales, M., Tapiadormorales, R., Gutierrezgalan, D., Cascadocaballero, D.: Wireless Sensor Network for Wildlife Tracking and Behavior Classification of Animals in Donãna. IEEE Communications Letters 20, 2534-2537 (2016)

5. Jaigirdar, F.T., Zullslam, M.M., Huq, S.R.: An Efficient and Cost Effective Maximum Clique Analysis based Approximation in Military Application of Wireless Sensor Network. International Conference on Computer \& Information Technology, pp. 85-90 (2011).

6. Rahman, M.U., Rahman, S., Mansoor, S., Deep, V., Aashkaar, M.: Implementation of ICT and Wireless Sensor Networks for Earthquake Alert and Disaster Management in Earthquake Prone Areas. Procedia Computer Science 85, 92-99 (2016)

7. Yang, T., Mu, D., Hu, W., Zhang, H.X.: Energy-efficient Border Intrusion Detection Using Wireless Sensors Network. EURASIP Journal on Wireless Communications and Networking 2014, 46-47 (2014)

8. Dai, H., Wu, X., Xu, L., Wu, F., Chen, G.: Practical Scheduling for Stochastic Event Capture in Energy Harvesting Sensor Networks. International Journal of Sensor Networks 18, 85-86 (2015)

9. Dai, H., Xu, L., Wu, X., Dong, C., Chen, G.: Impact of Mobility on Energy Provisioning in wireless Rechargeable Sensor Networks. Wireless Communications and Networking Conference (WCNC), pp. 20-21 (2013).

10. Zhu, X., Li, J., Zhou, M., Chen, X.: Optimal Deployment of Energy Harvesting Directional Sensor Networks for Target Coverage. IEEE Systems Journal 13, 377-388 (2019)

11. Yang, C., Chin, K.W.: On Nodes Placement in Energy Harvesting Wireless Sensor Networks for Coverage and Connectivity. IEEE Transactions on Industrial Informatics 13, 1-1 (2016)

12. Li, S., He, J., Zhang, X., Peng, J.: An Energy Efficient Multi-hop Charging Scheme with Mobile Charger for Wireless Rechargeable Sensor Network. International Conference on Algorithms and Architectures for Parallel Processing, pp. 45-46 (2015).

13. Chen, Y.C., Jiang, J.R.: Particle Swarm Optimization for Charger Deployment in Wireless Rechargeable Sensor Networks. International Conference on Computer \& Information Technology, pp. 231-236 (2016).

14. Yu, N., Dai, H., Liu, A.X., Tian, B.: Placement of Connected Wireless Chargers. IEEE INFOCOM, pp. 1-9 (2017).

15. Dai, H., Wang, X., Liu, A.X., Ma, H., Chen, G.: Optimizing Wireless Charger Placement for Directional Charging. IEEE Conference on Computer Communications, pp. 2-10 (2017).

16. Dai, H., Wang, X., Liu, A.X., Ma, H., Chen, G.: Robustly Safe Charging for Wireless Power Transfer. IEEE INFOCOM, pp. 1-1 (2018) 
17. Su, Y.X., Chi, R.: Multi-objective Particle Swarm-differential Evolution Algorithm. Neural Computing and Applications 28, 407-418 (2017)

18. Kiranyaz, S., Pulkkinen, J., Gabbouj, M.: Multi-dimensional particle swarm optimization for dynamic environments. International Conference on Innovations in Information Technology, pp. 34-38 (2008).

19. Civicioglu, P., Besdok, E.: A Conceptual Comparison of the Cuckoo-search, Particle Swarm Optimization Differential Evolution and Artificial Bee Colony Algorithms. Artif Intell Rev. 39, 315-46 (2013)

20. Mehrabian, A.R., Lucas, C.: A Novel Numerical Optimization Algorithm Inspired from Weed Colonization. Ecological Informatics 1, 35-66 (2006)

21. Mirjalili, S., Saremi, S., Mirjalili, S.M., Coelho, L.D.S.: Multi-objective Grey Wolf Optimizer: A Novel Algorithm for Multi-criterion Optimization. Expert Systems with Applications 47, 106-19 (2016)

22. Yang, X.S.: A New Metaheuristic Bat-Inspired Algorithm. Computer Knowledge \& Technology 284, 65-74 (2010)

23. Salgotra, R., Singh, U.: Application of Mutation Operators to Flower Pollination Algorithm. Expert Systems with Applications 79, 112-129 (2017)

24. Guo, P., Liu, X., Tang, T., Tang, S., Cao, J.: Practical Concurrent Wireless Charging Scheduling for Sensor Networks. IEEE International Conference on Distributed Computing Systems (ICDCS), pp. 741-742 (2016).

25. Xu, J., Yuan, X., Wei, Z., Han, J., Shi, L., Lyu, Z.: A Wireless Sensor Network Recharging Strategy by Balancing Lifespan of Sensor Nodes. IEEE Wireless Communications and Networking Conference (WCNC), pp. 1-6 (2017)

26. Chien, W., Cho, H., Chao, H., Shih, T.K.: Enhanced SA-based charging algorithm for WRSN. International Wireless Communications and Mobile Computing Conference (IWCMC), pp. 1012-1017 (2016).

27. Chen, Z., Shen, H., Zhao, X.: Delay-Tolerant On-Demand Mobile Charging Scheduling Scheme for Wireless Rechargeable Sensor Networks. International Symposium on Parallel Architectures, Algorithms and Programming (PAAP), pp. 29-35 (2018)

28. Tomar, A., Anwit, R., Jana, P.K.: An efficient scheme for on-demand energy replenishment in wireless rechargeable sensor networks. International Conference on Advances in Computing, Communications and Informatics (ICACCI), pp. 125-130 (2017).

29. Yang, M., Wang, A., Su, G., Zhang, Y.: Deploying Charging Nodes in Wireless Rechargeable Sensor Networks based on Improved Firefly Algorithm. Computers \& Electrical Engineering 6, 314-593 (2017)

30. Yang, X.S., Deb, S.: Multiobjective Cuckoo Search for Design optimization. Computes \& Operation Research 40, 1616-1624 (2013)

31. Shekhar, S., Chandra, C.: Optimal Control of A Service System with Emergency Vacation Using Bat Algorithm. Journal of computational and applied mathematics 2, 36-56 (2019) 